\title{
Backbone Dynamics Determined by Electron Paramagnetic Resonance to Optimize Solid-Phase Peptide Synthesis of TOAC-Labeled Phospholamban
}

\author{
Zhiwen Zhang, ${ }^{1}$ Henriette A. Remmer, ${ }^{2}$ David D. Thomas, ${ }^{1}$ Christine B. Karim ${ }^{1}$ \\ ${ }^{1}$ Department of Biochemistry, Molecular Biology and Biophysics, University of Minnesota, Minneapolis, MN 55455 \\ ${ }^{2}$ Department of Biological Chemistry, University of Michigan, Ann Arbor, MI 48109 \\ Received 30 May 2006; revised 13 October 2006; accepted 17 October 2006 \\ Published online 25 October 2006 in Wiley InterScience (www.interscience.wiley.com). DOI 10.1002/bip.20618
}

\begin{abstract}
:
Electron paramagnetic resonance (EPR) was used to optimize the solid-phase peptide synthesis of a membrane-bound peptide labeled with TOAC (2,2,6,6tetramethyl-piperidine-1-oxyl-4-amino-4-carboxylic acid). The incorporation of this paramagnetic amino acid results in a nitroxide spin label coupled rigidly to the $\alpha$-carbon, providing direct detection of peptide backbone dynamics by EPR. We applied this approach to phospholamban, which regulates cardiac calcium transport. The synthesis of this amphipathic 52-aminoacid membrane peptide including TOAC is a challenge, especially in the addition of TOAC and the next several amino acids. Therefore, EPR of synthetic intermediates, reconstituted into lipid bilayers, was used to ensure complete coupling and 9-fluorenylmethoxycarbonyl (Fmoc) deprotection. The attachment of Fmoc-TOAC$\mathrm{OH}$ leads to strong immobilization of the spin label, whereas Fmoc deprotection dramatically mobilizes it, producing an EPR spectral peak that is completely
\end{abstract}

Correspondence to: Christine B. Karim, Department of Biochemistry, Molecular Biology and Biophysics, University of Minnesota, Minneapolis, MN 55455; e-mail: cbk@ddt.biochem.umn.edu

Contract grant sponsor: National Institutes of Health Contract grant number: GM27906 Contract grant sponsor: American Heart Association Contract grant number: $9930083 \mathrm{~N}$

\section{InterScience}

C 2006 Wiley Periodicals, Inc. resolved from that observed before deprotection. Similarly, coupling of the next amino acid (Ser) restores the spin label to strong immobilization, giving a peak that is completely resolved from that of the preceding step. For several subsequent steps, the effect of coupling and deprotection is similar but less dramatic. Thus, the sensitivity and resolution of EPR provides a quantitative monitor of completion at each of these critical steps in peptide synthesis. Mass spectrometry, circular dichroism, and Edman degradation were used in concert with EPR to verify the chemistry and characterize the secondary structure. In conclusion, the application of conventional analytical methods in combination with EPR offers an improved approach to optimize the accurate synthesis of TOAC spin-labeled membrane peptides. (C) 2006 Wiley Periodicals, Inc. Biopolymers (Pept Sci) 88:29-35, 2007. Keywords: Edman sequencing; EPR; lipids; membrane protein; MS; phospholamban; reconstitution; solid-phase peptide synthesis; TOAC

This article was originally published online as an accepted preprint. The "Published Online" date corresponds to the preprint version. You can request a copy of the preprint by emailing the Biopolymers editorial office at biopolymers@wiley.com

\section{INTRODUCTION}

ite-directed spin-labeling is typically accomplished by producing a single-Cys mutant of a protein and then labeling with a thiol-specific spin label. The electron paramagnetic resonance (EPR) spectrum of a Cys-attached spin label is sensitive to the protein's 
secondary and tertiary structure near the labeled site, but flexible attachment of the probe makes the data only indirectly related to peptide backbone dynamics. ${ }^{1,2}$ This problem was solved by the introduction of the paramagnetic amino acid 2,2,6,6-tetramethylpiperidine-1-oxyl-4-amino-4-carboxylic acid (TOAC), which is rigidly coupled to the $\alpha$-carbon and thus reports more accurately the position, orientation, and dynamics of the peptide backbone. ${ }^{3-5}$ Using 9-fluorenylmethoxycarbonyl (Fmoc) methodology, ${ }^{6}$ TOAC can be inserted at any position in the peptide chain. ${ }^{7-9}$ Today the technology of stepwise Fmoc solid-phase peptide synthesis (SPPS) on a resin support is used routinely up to 50 residues in length, and optimized automated protocols have been described that extend target sequences to over 100 residues. $^{10}$

We previously reported the synthesis of phospholamban (PLB), ${ }^{11}$ a 52 -amino-acid integral membrane protein that regulates calcium transport in the heart. ${ }^{12}$ Although the spectroscopic techniques of NMR, ${ }^{13,14}$ FRET, ${ }^{15,16}$ FTIR, ${ }^{17}$ and circular dichroism $(\mathrm{CD})^{18,19}$ have been applied effectively to investigate PLB, spin labeling EPR has played a unique role in defining the functional dynamics of PLB's regulatory interaction with sarcoplasmic reticulum calcium ATPase (SERCA). ${ }^{11,13,20-22}$ SPPS allowed us to incorporate TOAC at selected sites. We then reconstituted labeled PLB into lipids, verified inhibitory function, and used EPR to determine the nanosecond rotational dynamics and membrane topology of four labeled sites in lipid membranes. ${ }^{11}$ Further studies with TOAC-PLB explored changes in structural dynamics upon phosphorylation and interaction of PLB with the regulatory target, SERCA. ${ }^{13,20}$ However, difficulty in coupling TOAC and subsequent amino acids has been a serious limitation of this spin probe. ${ }^{23}$ This is mostly due to the very low nucleophilicity of its amine function when bound to a peptide sequence $\left(\mathrm{p} K_{\mathrm{a}}<6\right) .^{24,25}$ This problem can be overcome when a more efficient coupling procedure is applied. ${ }^{9}$ The synthesis and analysis of the 52 amino acid membrane peptide represents an additional challenge. The hydrophobic residues in the transmembrane domain of PLB can lead to steric hindrance and inaccessibility of the free amino group to the growing peptide chain. Side-products that build up over many coupling steps often make purification difficult. Because of these problems, the successful TOAC-PLB synthesis was found to require further analytical optimization, with a combination of analytical methods that can enhance quality assurance throughout the synthetic process.

The primary innovation in the present study is the use of EPR spectroscopy to provide detailed analysis of the chemical structure at key points in the synthetic procedure. MALDITOFMS of the peptide segments was used before TOAC coupling. EPR of lipid-bound synthetic intermediates was used

\section{${ }^{11} \tau$ IRRASTIEMPQQARQNLQNLFINFALILIFLLLIAIIVMLL ${ }^{52}$}

FIGURE 1 Amino acid sequence from 11 to 52 of AFA-PLB. $\operatorname{TOAC}(\tau)$ is substituted for Ala at position 11.

to monitor Fmoc-TOAC coupling and Fmoc deprotection. CD spectroscopy was used to detect changes in secondary structure, thus aiding in the interpretation of EPR spectra.

\section{MATERIALS AND METHODS}

\section{Solid-Phase Peptide Synthesis of TOAC-PLB}

Figure 1 shows the sequence of the PLB segment from amino acid 11-52, with the spin-labeled amino acid TOAC attached at position 11. Protocols for the PLB solid-phase peptide synthesis, deprotection, cleavage, and HPLC purification, ${ }^{19,26,27}$ 9-fluorenylmethoxycarbonyl (Fmoc)-TOAC-OH synthesis, ${ }^{5,9}$ and TOAC attachment at position 11 on $\mathrm{PLB}^{11,20}$ have been reported previously. We showed that monomeric AFA-PLB has similar inhibitory activity as wildtype PLB, and that AFA-PLB retains inhibitory potency when Ala11 is replaced by TOAC. $^{11}$ EPR spectra of TOAC at this labeling site have been shown to be quite sensitive to functionally important structural changes in the cytoplasmic domain of AFA-PLB. ${ }^{20}$

\section{Liposome Preparation}

DOPC and DOPE phospholipids were obtained from Avanti Polar Lipids. Lipids were dissolved in chloroform and peptide in methanol. These solutions were mixed, with a molar ratio of 200 lipids per peptide, in a glass tube and dried under a low flow of nitrogen to form a thin film. Residual solvent was removed under vacuum for several hours. The resulting lipid film was hydrated with an appropriate volume of $10 \mathrm{mM}$ Tris ( $\mathrm{pH} 7.0$ ), to yield a final lipid concentration of 8-10 $\mathrm{mM}$, and vortexed to produce lipid vesicles. Vesicle suspensions for circular dichroism (CD) experiments were sonicated $20 \mathrm{~min}$ or more in a bath sonicator. This process produced clear solutions that scattered light only minimally in the 190 $250 \mathrm{~nm}$ wavelength range used for CD.

\section{CD}

CD spectra were recorded on a Jasco J-710 spectrophotometer at $25^{\circ} \mathrm{C}$ using a $0.01 \mathrm{~cm}$ path-length quartz cuvette. Acquisition was performed using a $50 \mathrm{~nm} / \mathrm{min}$ scan rate, $1 \mathrm{~nm}$ bandwidth, and $2 \mathrm{~s}$ filter time constant. The corresponding baseline (buffer or lipid/ buffer solution) was subtracted from each spectrum. Each reported spectrum is the average of six scans, expressed as mean residue ellipticity, $[\theta]$. Aqueous solutions for $\mathrm{CD}$ were prepared by drying a volume of peptide stock solution $(\sim 3 \mathrm{mg} / \mathrm{ml}$ in chloroform or methanol) under a low flow of $\mathrm{N}_{2}$ and adding the appropriate amount of $10 \mathrm{mM}$ Tris ( $\mathrm{pH} 7.0$ ) to give final peptide concentrations of $0.1-$ $0.5 \mathrm{mM}$. CD basis spectra corresponding to pure $\alpha$-helix, $\beta$-sheet, or random coil were obtained from standard samples of poly(lysine) and poly(glutamic acid) (Sigma), using conditions and parameters reported previously. ${ }^{28,29}$ Each spectrum was fit by a linear combination of these basis spectra to determine the mole fraction of amino acids contributing to each type of secondary structure. ${ }^{19}$ 


\section{MALDI-TOF-MS}

Mass spectral data was acquired with a Bruker Biflex III MatrixAssisted Laser Desorption/Ionization Time-of-Flight (MALDITOF) system, which is equipped with an $\mathrm{N}_{2}$-laser (337 nm, 3-nanosecond pulse length) and a microchannel plate detector. The data was collected in the linear mode, positive polarity, with an accelerating potential of $19 \mathrm{kV}$. Each spectrum is the accumulation of 100 400 laser shots. The samples were cocrystallized with the matrix 3,5dimethoxy-4-hydroxycinnamic acid (sinapinic acid).

\section{EPR Spectroscopy}

For EPR experiments, PLB was reconstituted into lipid vesicles containing DOPC/DOPE (4:1, 200 lipids per PLB) in $25 \mathrm{mM}$ imidazole ( $\mathrm{pH}$ 7.0). ${ }^{11}$ Scan width $120-\mathrm{G}$ spectra were acquired using a Bruker EleXsys 500 spectrometer equipped with the SHQ cavity, using $100 \mathrm{kHz}$ field modulation with peak-to-peak amplitude of $3.0 \mathrm{G}$ and microwave power of $12.6 \mathrm{~mW}$, corresponding approximately to the maximum signal. ${ }^{11,20}$ A typical sample contained $5 \mu \mathrm{l}$ of $0.5 \mathrm{mM}$ PLB, loaded into a $25 \mu \mathrm{l}$ Wiretrol (Drummond Scientific Company). Sample temperature was maintained at $4^{\circ} \mathrm{C}$ using the Bruker temperature controller, with the sample cell inside a quartz Dewar.

\section{Protein Sequencing}

$\mathrm{N}$-terminal amino acid sequencing on resin-bound full-length crude PLB was performed by automated Edman sequencing using an Applied Biosystems 494HT sequencer equipped with a $610 \mathrm{~A}$ data analysis module (version 2.1). All operations were carried out as prescribed in the Applied Biosystems sequencer manual.

\section{RESULTS}

The combined analytical approach was carried out as follows: (1) preliminary analysis of the crude PLB12-52 segment by MALDI/TOFMS, (2) EPR to monitor Fmoc TOAC coupling and Fmoc deprotection, (3) CD of the purified PLB segments to determine secondary structural composition, and (4) Edman sequencing to confirm the final PLB sequence.

\section{Analysis by MALDI/TOFMS}

MALDI/TOFMS of the crude PLB segment synthesized from amino acid 12-52 confirmed its high purity. The mass spectrum (Figure 2) shows the presence of one main peak corresponding to $\mathrm{m} / z$ value of $4767.6[\mathrm{M}+\mathrm{H}]^{+}$, which is in agreement with the theoretical value of the PLB12-52 segment of 4766.8 Da. The minor peak at an $m / z$ value of $4654.5[\mathrm{M}+$ $\mathrm{H}]^{+}$indicates the presence of impurity, which could be ascribed to the deletion of one leucine or isoleucine. The purity was increased after HPLC purification, and no $\mathrm{N}_{\alpha^{-}}$ Fmoc-terminated peptides were detected by mass spectroscopy.

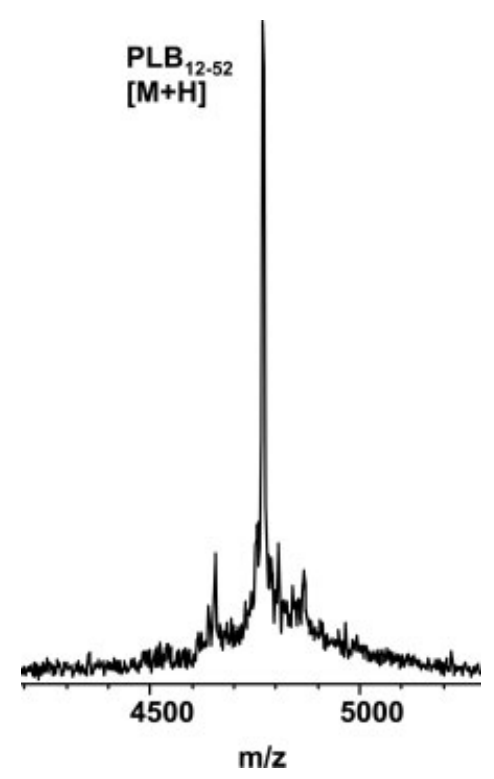

FIGURE 2 Positive-mode linear MALDI mass spectrum of crude PLB segment 12-52.

\section{Detection of Peptide Dynamics by EPR}

The Fmoc group (structure in Figure 3, top, right) is the most widely used group in urethane-type protection. ${ }^{6,30,31}$ The geometry of the Fmoc group is rigid, with the fluorenyl ring unchanged with respect to the unsubstituted fluorene molecule. ${ }^{32}$ Coupling such $\mathrm{N}_{\alpha}$-protected amino acids with other amino acids or peptides proceeds without racemization, whatever activation method is employed. ${ }^{33}$ Side chain protection of Fmoc amino acids that can undergo irreversible side reactions during SPPS require blocking groups that are ideally base stable and TFA labile. ${ }^{34}$ For PLB synthesis, the acidolyzable side chain protecting groups have been recently published. ${ }^{27}$ Serine has been protected by a tBu side chain to avoid active ester coupling of unprotected FmocSer during SPPS. Similarly, to prevent acetylation of Arg, it was protected by a Pmc side chain. ${ }^{34}$ The aim of these experiments was to use EPR to monitor structural changes that occur during Fmoc coupling and deprotection in the synthesis of TOAC-spin-labeled PLB. Samples for EPR were obtained after the final cleavage from the resin with TFA, which also removes the side chain protecting groups. Then each crude PLB segment was reconstituted in lipid bilayers, and the EPR spectrum was recorded. Thus in the pairs of spectra in Figure 3 , the only difference is whether the Fmoc group remains on the $\mathrm{N}$ terminus.

Figure 3 shows EPR spectra upon the coupling of FmocTOAC at position 11 on PLB, followed by Fmoc deprotection and subsequent steps in the peptide chain assembly from position 11-9. In each case, the removal of the Fmoc group (deprotection) greatly increases the mobility of the TOAC 


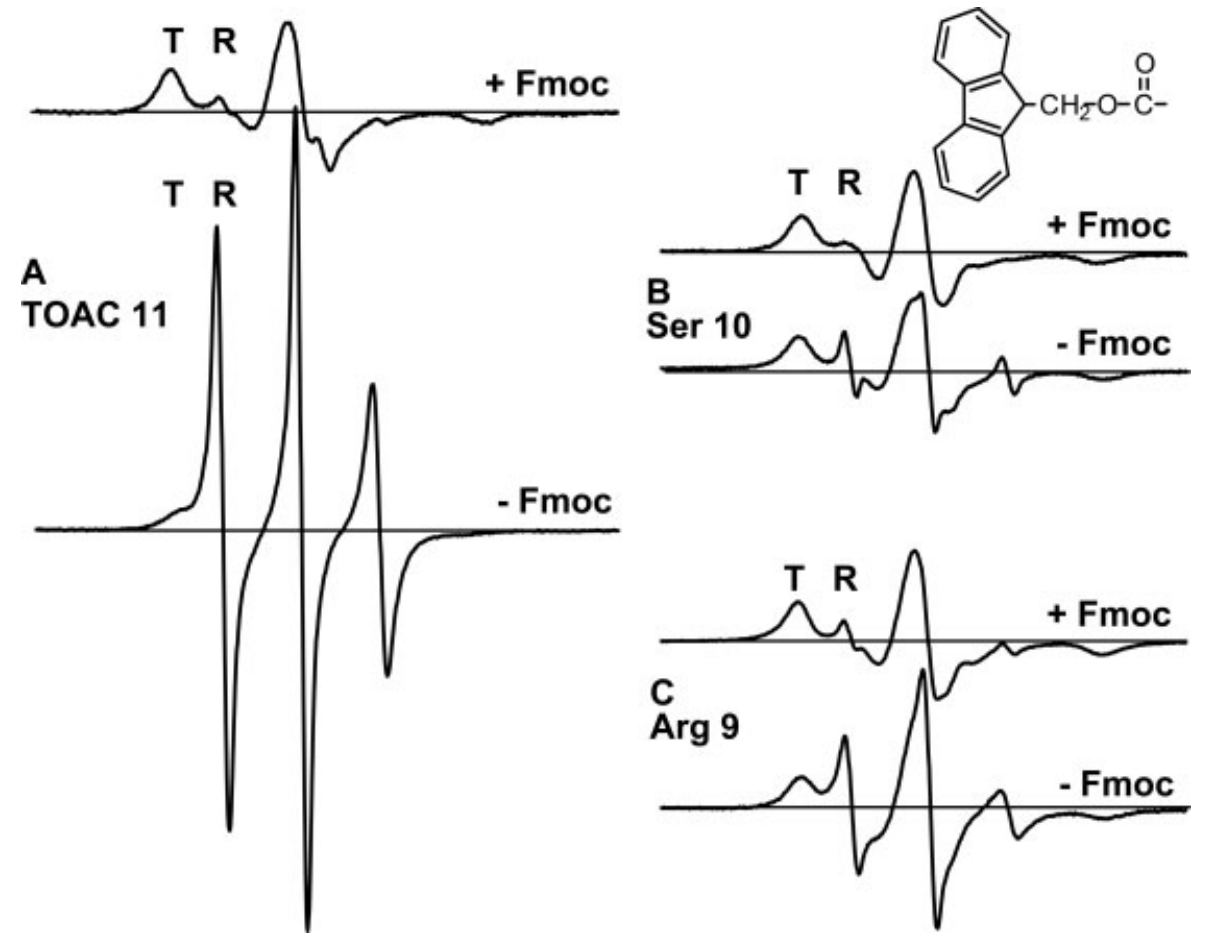

FIGURE 3 EPR spectra of sequential synthetic intermediates in lipid bilayers. Each pair of spectra was obtained before and after Fmoc removal; (A) PLB12-52 after coupling of FmocTOAC at position 11, (B) after subsequent coupling of FmocSer at position 10, and (C) after subsequent coupling of FmocArg at position 9. Spectra were normalized to unit concentration by dividing by the double integral. Scan width 120-G.

spin label at position 11 (see Figure 3), i.e., each "Fmoc" spectrum contains a larger contribution from peak $T$ (corresponding to an ordered $\alpha$-helical conformation of the peptide backbone) than its corresponding deprotected ("-Fmoc") spectrum. In particular, Fmoc deprotection of TOAC 11 almost completely eliminates the ordered population $T$ (Figure 3A, bottom), in favor of the dynamically disordered population that gives rise to peak $R$.

The subsequent coupling of FmocSer at position 10 has an equally significant effect in the opposite direction, completely immobilizing the spin label at TOAC 11, so that only the $T$ population is observed (Figure 3B, top). This pattern continues, in less significant fashion, for the next Fmoc protected amino acid (Arg 9), as the peptide synthesis continues (Figure 3C).

The hydrophobic nature of the attached Fmoc group probably interacts with the hydrophobic phase of the lipid bilayer, inducing a more stable helix within this N-terminal region, which causes the restriction in nanosecond dynamics.

\section{CD}

To more fully characterize the PLB system, we used CD spectroscopy to measure the secondary structure of the PLB seg- ments studied by EPR Figure 3. The CD spectrum of FmocPLB12-52 mixed with DOPC/DOPE (50:1) lipids shows the characteristic $\alpha$-helical negative maxima at 207 and $222 \mathrm{~nm}$ and positive maximum at $193 \mathrm{~nm}$ (Figure 4, red). The linear fit of the spectrum (CD data analysis reported in Material and Methods section) yielded $85-90 \%$ helix. After Fmocdeprotection (Figure 4, black), the spectrum differs substantially, suggesting a partial loss of $\alpha$-helical structure. This supports the hypothesis that the Fmoc group stabilizes the helix within the $\mathrm{N}$-terminal region of the peptide, because of its interaction with the lipid bilayer membrane. This structural enhancement could arise from interpeptide interactions ${ }^{19}$ or interactions between the fluorenyl groups. ${ }^{35}$

\section{Protein Sequencing}

Automated Edman sequencing (see Figure 5) was used to confirm the primary structure of the completed peptide 11TOAC-AFA-PLB (sequence of full-length AFA-PLB Figure 6 , top). Although mass spectrometry is an efficient method for determining peptide sequence, it has limitations. The insertion of unnatural amino acids or free radicals generates ambiguity. Furthermore, MS analysis requires the additional 


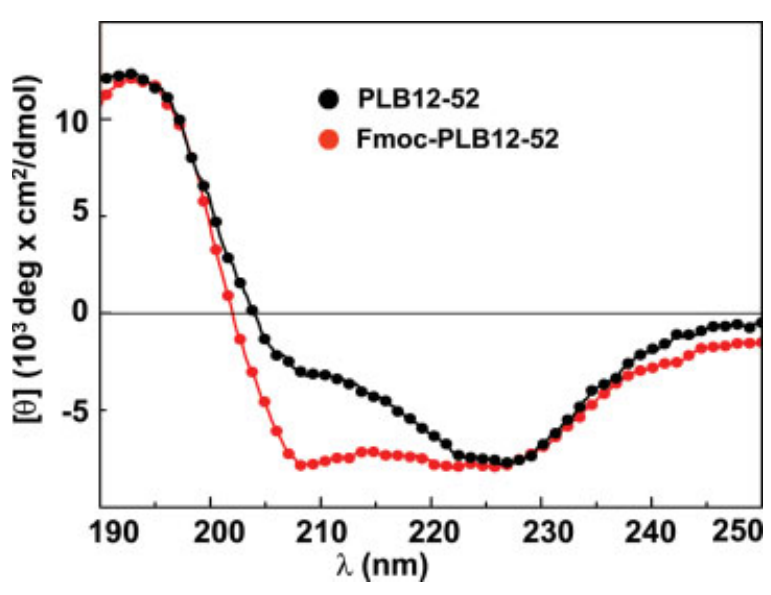

FIGURE 4 CD spectra of Fmoc protected PLB12-52 (red) and after Fmoc deprotection in DOPC/DOPE mixture (50:1 lipid:peptide) $10 \mathrm{mM}$ Tris, $\mathrm{pH} 7.0,25^{\circ} \mathrm{C}$.

steps of peptide cleavage and extraction from the resin. Edman degradation sequence analysis can be used for evaluating the synthesis for resin-bound peptides. ${ }^{36}$ Edman chemistry converts each $\alpha$-amino acid sequentially to its PTH derivative. The formed PTH amino acids can be identified by their retention times on HPLC by comparison with reference standards. Since the by-product DPTU appears on each amino acid residue cycle during the process of sequencing, this peak can be used as excellent internal reference peak. ${ }^{37}$ Edman sequencing of peptides containing unnatural amino acids was previously reported, ${ }^{37}$ but there is no literature on the sequencing of TOAC peptides. We used automated Edman sequencing to confirm the final 10 amino acids after TOAC coupling at position 11. Figure 5A shows the chromatography of the standard mixture of the $19 \mathrm{PTH}$ amino acids used as reference for the amino acid assignment. Figures 5B5D shows the HPLC elution profile of the first identified amino acid Met 1, as well as the last two Arg 9 and Ser 10 . The sequence analysis confirmed the successful synthesis of the full-length AFA-PLB with TOAC inserted at position 11.

\section{Accessibility to the Membrane Surface}

To verify the interaction of the Fmoc group with the lipid membrane, EPR experiments were performed on full-length AFA-PLB (Figure 6, top) with TOAC attached to the N terminus. The EPR spectra (Figure 6, left) show that the Fmoc group stabilizes the restricted ("T") component, just as it does in the intermediate segment PLB11-52. Power saturation was used to measure the accessibility of the spin label to $\mathrm{Ni}^{2+}$ ions chelated to the lipid headgroup. Figure 6, right shows that accessibility of the spin-labeled peptide to the membrane surface was higher before the removal of Fmoc (black) than after (grey), confirming that the Fmoc group anchors the $\mathrm{N}$-terminus to the lipid bilayer.

\section{DISCUSSION}

In solid-phase peptide synthesis, Fmoc has become the most frequently used protecting group. ${ }^{6,38}$ Its utility for the synthesis of wide varieties of peptide conjugates, including Fmoc-TOAC derivatives of membrane proteins, has been reported. ${ }^{11,20}$ In the present study, EPR of lipid-bound synthetic intermediates was applied to monitor Fmoc-TOAC coupling and deprotection. The EPR spectra in Figure 3 clearly show that Fmoc deprotection increases TOAC mobility, and coupling of the next Fmoc amino acid decreases

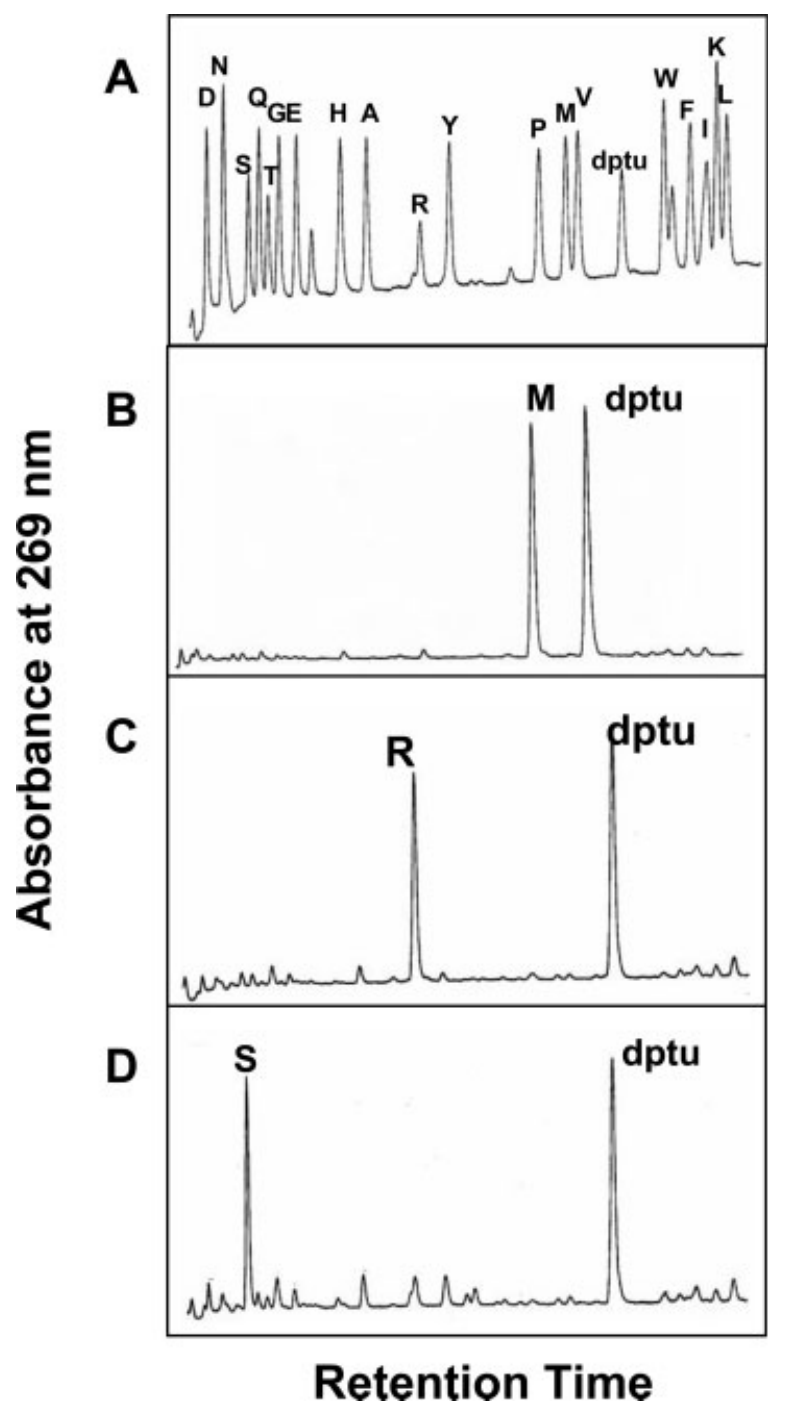

FIGURE 5 Elution profiles of $19 \mathrm{PTH}$ amino acids (A), Met 1 (B), Arg 9 (C), and Ser 10 (D). 

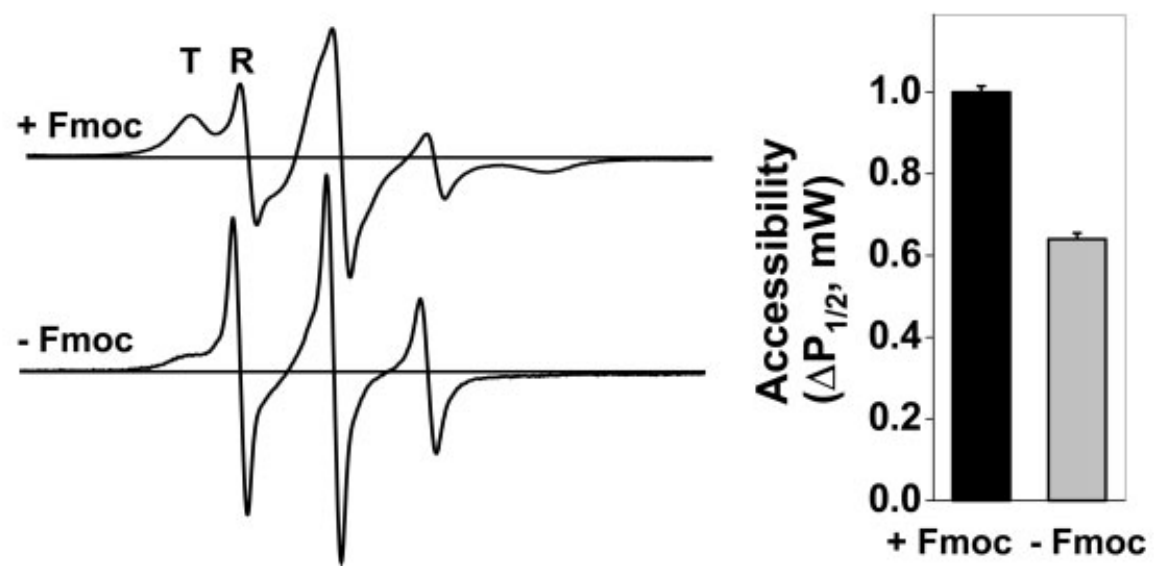

FIGURE 6 EPR spectra showing that the Fmoc group increases interaction of the PLB N-terminus with the lipid bilayer. Top: Sequence of full-length AFA-PLB with TOAC $(\tau)$ attached at the Nterminus. Left: EPR spectra of N-terminal Fmoc-TOAC-AFA-PLB, reconstituted into lipid bilayers, before $(+$ Fmoc $)$ and after $(-$ Fmoc $)$ deprotection. Spectra were normalized to unit concentration by dividing by the double integral. Scan width $120-\mathrm{G}$ at $4^{\circ} \mathrm{C}$. Right: Accessibility to the membrane surface of TOAC before (black) and after (grey) Fmoc deprotection. The accessibility (normalized to 1 for + Fmoc) was measured as the increase in microwave power needed to for half-saturation of the EPR signal, upon addition of $\mathrm{Ni}^{2+}$ chelated to lipid head groups, as described previously. ${ }^{11}$

TOAC mobility. Most importantly, the high resolution of EPR provides quantitative measurement of the efficiencies of several of the steps. Note that the $R$ component of the EPR spectrum has negligible intensity before deprotection, and the $T$ component has negligible intensity after deprotection (Figure 3A). This shows clearly that the efficiency of deprotection is essentially $100 \%$ and that anything less than $100 \%$ deprotection would be detected quantitatively as a peak at the $T$ position. Similarly, in the next step, the coupling of FmocSer to position 10 , the $R$ component is completely eliminated showing that this step has gone to completion as well. Thus, anything less than $100 \%$ coupling at this step would be detected quantitatively as a peak at the $R$ position. In the subsequent steps, the spectral changes are not so extreme, so the EPR spectra do not provide such a quantitative monitor of the reaction.

The EPR spectra correlated well with conformational changes obtained by CD. The observed decrease in the ordered $T$ component in EPR upon deprotection (Figure $3 \mathrm{~A}$ ) corresponds to a decrease in $\alpha$-helical content (see Figure 4). EPR data in Figure 6 confirms that the rigid hydrophobic Fmoc group anchors the cytoplasmic domain to the lipid bilayer membrane by hydrophobic interactions and stabilizes the helical structure. These results are consistent with a previous study in which a lipid group attached to the $\mathrm{N}$ terminus of PLB increased the interaction of the N-terminal domain of PLB with the lipid surface and increased helical order in this domain. ${ }^{20}$ Crystal structural studies of Fmoc derivatives suggest that its amide $-\mathrm{CONH}-$ and the urethane $\mathrm{C}=\mathrm{O}$ group interact with the solvent molecules and stabilize the helix. ${ }^{33}$ Previous structure-activity investigations on angiotensin analogs containing the bulky, lipophilic substituent Fmoc at the N-terminus induce tachyphylaxis, defined as the acute loss of response of some smooth muscles. The Fmoc affinity for the lipid membrane induces structural changes in the peptide. ${ }^{39}$

In conclusion, the results show that the application of conventional analytical methods in combination with EPR offers an improved approach to optimize the accurate synthesis of spin-labeled membrane peptides. The EPR studies in lipids show that the attachment of Fmoc-TOAC leads to strong immobilization, whereas Fmoc deprotection mobilizes the probe. CD data confirm the conformational changes: Fmoc induces a more stable helix within this N-terminal region of the peptide by hydrophobic interaction with the membrane surface. EPR is of great value in monitoring coupling and deprotection steps during solid phase peptide synthesis of membrane peptides since it provides relevant information about conformational changes induced by the Fmoc group. This approach is applicable to a large number of small membrane proteins that, like PLB, have transmembrane helices coupled to short cytoplasmic regulatory domains, such as sarcolipin $^{40}$ and the FXYD family of plasma membrane regulators. $^{41}$ 
We thank Yuri Nesmelov for assistance with EPR spectroscopy, Sudha Marimanikkuppam and Thomas Krick for assistance with mass spectrometry (University of Minnesota, Minneapolis, MN 55455). We thank Jinny Johnson and Lawrence Dangott for amino acid analysis (Protein Chemistry Laboratory, Texas A\&M University, College Station, TX 77843).

\section{REFERENCES}

1. Chen, B.; Bigelow, D. J. Biochemistry 2002, 41, 1396513972.

2. Hubbell, W. L.; Cafiso, D. S.; Altenbach, C. Nat Struct Biol 2000, 7, 735-739.

3. Monaco, V.; Formaggio, F.; Crisma, M.; Toniolo, C.; Hanson, P.; Millhauser, G.; George, C.; Deschamps, J. R.; Flippen-Anderson, J. L. Bioorg Med Chem 1999, 7, 119-131.

4. Monaco, V.; Formaggio, F.; Crisma, M.; Toniolo, C.; Hanson, P.; Millhauser, G. L. Biopolymers 1999, 50, 239-253.

5. Toniolo, C.; Valente, E.; Formaggio, F.; Crisma, M.; Pilloni, G.; Corvaja, C.; Toffoletti, A.; Martinez, G. V.; Hanson, M. P.; Millhauser, G. L.; George, C.; Flippen-Anderson, J. L. J Pept Sci 1995, 1, 45-57.

6. Atherton, E.; Sheppard, R. C. Solid Phase Peptide Synthesis. A Practical Approach; IRL Press: Oxford, 1989.

7. Martin, L.; Ivancich, A.; Vita, C.; Formaggio, F.; Toniolo, A. Pept Res 2001, 58, 424-432.

8. Hanson, P.; Martinez, G. V.; Millhauser, G. L.; Formaggio, F.; Crisma, M.; Toniolo, C.; Vita, C. J Am Chem Soc 1996, 118, 271-272.

9. Marchetto, R.; Schreier, S.; Nakaie, C. R. J Am Chem Soc 1993 , 115, 11042, 11043.

10. Muir, T. W.; Kent, S. B. Curr Opin Biotechnol 1993, 4, 420427.

11. Karim, C. B.; Kirby, T. L.; Zhang, Z.; Nesmelov, Y.; Thomas, D. D. Proc Natl Acad Sci USA 2004, 101, 14437-14442.

12. Lindemann, J. P.; Jones, L. R.; Hathaway, D. R.; Henry, B. G.; Watanabe, A. M. J Biol Chem 1983, 258, 464-471.

13. Zamoon, J.; Nitu, F.; Karim, C.; Thomas, D. D.; Veglia, G. Proc Natl Acad Sci USA 2005, 102, 4747-4752.

14. Metcalfe, E. E.; Traaseth, N. J.; Veglia, G. Biochemistry 2005, 44, 4386-4396.

15. Robia, S. L.; Flohr, N. C.; Thomas, D. D. Biochemistry 2005, 44, 4302-4311.

16. Mueller, B.; Karim, C. B.; Negrashov, I. V.; Kutchai, H.; Thomas, D. D. Biochemistry 2004, 43, 8754-8765.
17. Tatulian, S. A.; Chen, B.; Li, J.; Negash, S.; Middaugh, C. R.; Bigelow, D. J.; Squier, T. C. Biochemistry 2002, 41, 741-751.

18. Paterlini, M. G.; Thomas, D. D. Biophys J 2005, 88, 3243-3251.

19. Lockwood, N. A.; Tu, R. S.; Zhang, Z.; Tirrell, M. V.; Thomas, D. D.; Karim, C. B. Biopolymers 2003, 69, 283-292.

20. Karim, C. B.; Zhang, Z.; Howard, E. C.; Torgersen, K. D.; Thomas, D. D. J Mol Biol 2006, 358, 1032-1040.

21. Kirby, T. L.; Karim, C. B.; Thomas, D. D. Biochemistry 2004, 43, 5842-5852.

22. Negash, S.; Yao, Q.; Sun, H.; Li, J.; Bigelow, D. J.; Squier, T. C. Biochem J 2000, 351, 195-205.

23. Tominaga, M.; Barbosa, S. R.; Poletti, E. F.; Zukerman-Schpector, J.; Marchetto, R.; Schreier, S.; Paiva, A. C.; Nakaie, C. R. Chem Pharm Bull (Tokyo) 2001, 49, 1027-1029.

24. Nakaie, C. R.; Goissis, G.; Schreier, S.; Paiva, A. C. Braz J Med Biol Res 1981, 14, 173-180.

25. Nakaie, C. R.; Schreier, S.; Paiva, A. C. Biochim Biophys Acta 1983, 742, 63-71.

26. Karim, C. B.; Paterlini, M. G.; Reddy, L. G.; Hunter, G. W.; Barany, G.; Thomas, D. D. J Biol Chem 2001, 26, 26.

27. Karim, C. B.; Marquardt, C. G.; Stamm, J. D.; Barany, G.; Thomas, D. D. Biochemistry 2000, 39, 10892-10897.

28. Greenfield, N.; Fasman, G. D. Biochemistry 1969, 8, 4108-4116.

29. Adler, A. J.; Greenfield, N. J.; Fasman, G. D. Methods Enzymol 1973, 27, 675-735.

30. Carpino, L. A.; Han, G. Y. J Org Chem 1972, 37, 3404-3409.

31. Carpino, L. A.; Han, G. Y. J Am Chem Soc 1970, 92, 5748, 5749.

32. Naider, F.; Goodman, M. In Synthesis of Peptides and Peptidomimetics (Houben-Weyl Methods of Organic Chemistry); Goodman, M.; Felix, A.; Moroder, L.; Tonido, C., Eds.; Thieme: Stuttgart, Germany, 2002; Vol. 22a, pp 1-16.

33. Broda, M. A.; Mazur, L.; Koziol, A. E.; Rzeszotarska, B. J Pept Sci 2004, 10, 448-461.

34. Fields, G. B.; Noble, R. L. Int J Pept Protein Res 1990, 35, 161-214.

35. Yang, Z.; Gu, H.; Zhang, Y.; Wang, L.; Xu, B. Chem Commun (Camb) 2004, 208-209.

36. Fields, C. G.; VanDrisse, V. L.; Fields, G. B. Pept Res 1993, 6, 39-47.

37. Liu, R.; Lam, K. S. Anal Biochem 2001, 295, 9-16.

38. Sheppard, R. J Pept Sci 2003, 9, 545-552.

39. Motta, S. C.; Poletti, E. F.; Souza, S. E.; Correa, S. A.; Jubilut, G. N.; Paiva, A. C.; Shimuta, S. I.; Nakaie, C. R. J Pept Res 2003, $62,227-232$.

40. Buffy, J. J.; Buck-Koehntop, B. A.; Porcelli, F.; Traaseth, N. J.; Thomas, D. D.; Veglia, G. J Mol Biol 2006, 358, 420-429.

41. Garty, H.; Karlish, S. J. Annu Rev Physiol 2006, 68, 431-459. 